\section{§5. Effect of Surface Angle on Mechanical Jointing of HTS}

\section{Hashizume, H., I to, S., Y amamoto, Y . (Tohoku U niv.)} Sagara, A. (NIFS)

For realization of commercial fusion power plant, remountable HTS (high-temperature superconductor) magnet is proposed to reduce both construction and maintenance costs [1]. The remountable HTS magnet can make it possible to simplify fabrication of reactor and facilitate access into inner structural components. In addition, when electromagnetic force produced by self magnetic field of HTS magnet can be used as joint force, there is possibility to mount and demount parts of the remountable HTS magnet easily.

In this study, the joint resistance in the butt joint of the BSCCO 2223 cable which has variable angle of the joint surface is evaluated experimentally to investigate the influence of shear stress on the resistance and clarify optimum joint condition.

10 ply BSCCO 2223 cable is used as a test cable in this experiment. Specifications of the BSCCO 2223 tape used for the cable are as follows. Critical current is $90 \mathrm{~A}$ under liquid nitrogen temperature $77 \mathrm{~K}$ with self magnetic field. The tape sizes are $4.2 \mathrm{~mm}$ wide and $0.21 \mathrm{~mm}$ thick. The joint surface of cable is cut down by angle of 30,40 , 45, 50 and 60 degrees inclined from length direction of the cable. Figure 2 shows the experimental system with the samples. Normal and shear stresses on the joint surface is loaded by compressing the cable with a rod in perpendicular direction of the cable as shown in Figure 1. The joint resistance of each sample is evaluated with changing the stress acting on the joint surface.

Results obtained through this experiment are shown in Figure 2. Figure 2(a) shows relationship between the normal stress and normalized joint resistance. The joint resistance decreases with increase of the normal stress in each case. Values of the normalized resistance are almost the same when the joint surface angles are 30,40 and 45 degrees. In case of 50 and 60 degrees, however, the normalized resistance increases with increase of the angles. This result implies that the joint resistance is not inversely proportional to joint area when the angle of the joint surface becomes larger than 50 degrees. Figure 3(b) shows relationship between the shear stress and normalized joint resistance when the normal stress is $62 \mathrm{MPa}$ and $101 \mathrm{MPa}$. When the shear stress is smaller than the normal stress, the values of normalized resistance are almost constant, while the shear stress becomes larger than the normal stress, the joint resistance shows larger value with increasing the shear stress. It is considered that the shear stress larger than the normal stress degrades the joint performance due to displacement of the joint surface. Consequently the joint performance is improved by forming the large joint area with surface configuration to give the most suitable stress distribution where the normal stress itself is large and the shear stress is smaller than the normal stress.
In this study, the joint resistance is evaluated in the butt joint of the BSCCO 2223 cable with changing angle of the joint surface. From experimental results, the joint performance varies with changing the joint area and stress distribution on the joint surface. Therefore, in designing phase of the remountable HTS magnet, it becomes important to form the joint surface in order to obtain the optimum stress distribution with the large normal and small shear stresses to achieve better butt joint performance

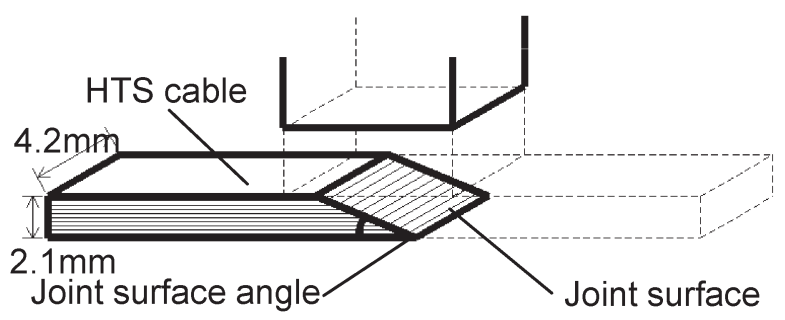

Figure 1: Experimental system.

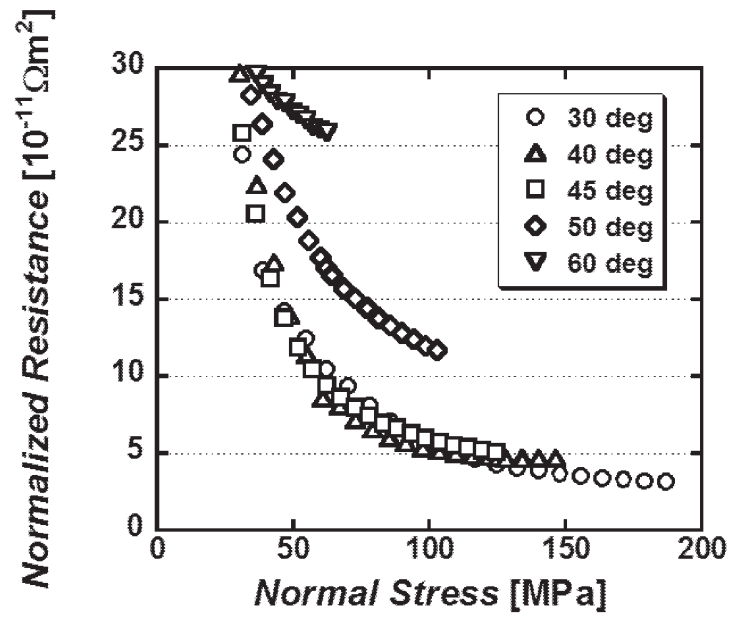

(a) Normal stress and resistance characteristic

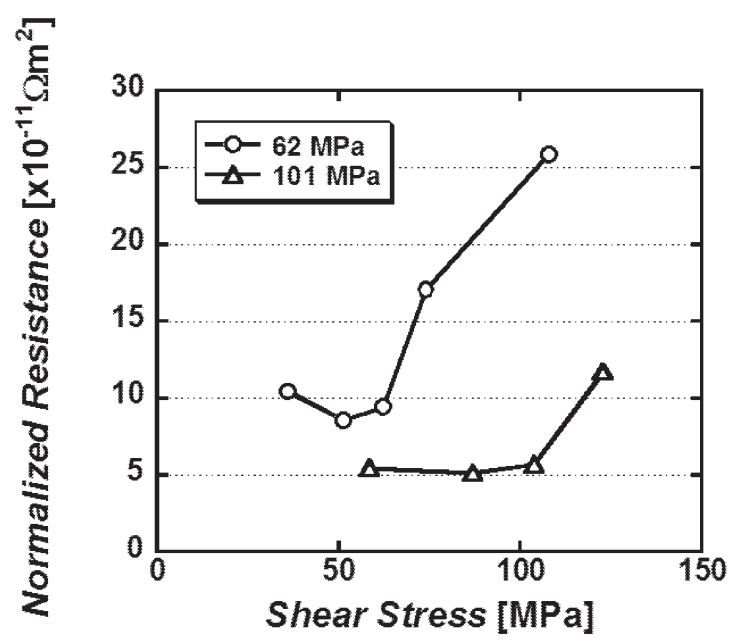

(b) Shear stress and resistance characteristic Figure 2: Experimental results 